Proposed upper limits for maximal LVEDD for white male is $<60 \mathrm{~mm}$ and for black and mixed-race males $<59 \mathrm{~mm}$. For all females the cut-off is $<52 \mathrm{~mm}$.

Conclusions Black athletes of both sexes reveal the greatest wall thickness compared with mixedrace and white athletes. Conversely, white athletes display the largest cavity size in both sexes. Mixed-race athletes demonstrate electrocardiogram and echocardiogram features more similar to black athletes than white athletes. Information regarding the upper limits of normality in this age group is useful in enabling the differentiation between physiological adaptation and cardiomyopathy.

\section{P62 DEFICIENCY PROTECTS AGAINST CEREBRAL ISCHAEMIA IN INSULIN RESISTANT MICE}

Siddall, Keith Farrell-Dillon, Paul Fraser, Giovanni Mann, Sarah Chapple. King's College London, UK

10.1136/postgradmedj-2018-fpm.20

Introduction and aim Diabetic patients have poorer outcomes following ischaemic stroke. In rodents, similar findings are reported, with Type 1 and 2 diabetic rodents demonstrating increased infarct volume and worsened post-stroke recovery, which correlate with markers of autophagy overactivation post-ischaemia. p62 is a selective adaptor protein for autophagy, with mice lacking p62 (p62 KO) known to develop obesity-linked insulin resistance in adulthood and have supressed autophagic flux. To ascertain whether p62 is associated with ischaemic stroke pathophysiology, we evaluated post-stroke infarct volume and functional recovery in wild type (WT) vs p62 KO mice.

Methods All procedures were performed in male 8-9 week old C57BL/6 mice in accordance with the ARRIVE guidelines and Home Office regulatory approval. Transient focal ischaemia was induced by middle cerebral artery occlusion (MCAo) for 1 hour followed by reperfusion for 72 hour. Adhesive removal and open field assays were conducted daily 3 days prior to and 72 hour post-stroke to assess sensorimotor and motor function. Mice were then euthanised and brains perfused-fixed to obtain $10 \mu \mathrm{m}$ sections every $1 \mathrm{~mm}$ relative to bregma. Infarct volume and astrocytosis were quantified using nissl staining and GFAP immunofluorescence, respectively. Data were analysed by unpaired student's t-test or 2-way ANOVA and Bonferroni post-hoc test as appropriate.

Results Infarct volume assessed by nissl staining showed a significant $(\mathrm{p}<0.01)$ reduction in $\mathrm{p} 62 \mathrm{KO}$ vs WT mice $(16 \pm 3$ vs. $30 \% \pm 1 \%$ brain volume, respectively). Proliferation and activation of astrocytes assessed by GFAP staining was found to be reduced in the peri-infarct border in p62 KO vs WT mice $(\mathrm{p}<0.05)$. Furthermore, p62 KO showed marked improvements in post-reperfusion functional recovery. Reduced motor function was observed in WT mice, with a $70 \%$ reduction in max speed and total displacement vs baseline observed 72 hour post-reperfusion $(\mathrm{p}<0.01)$. No such impairments were reported in p62 KO mice. Similarly, sensorimotor function was impaired in WT mice post-reperfusion, with an increase in adhesive removal time in the affected $(227 \pm 32$ vs $29 \pm 9 \mathrm{~ms}$ at baseline, $\mathrm{p}<0.001)$ and unaffected $(73 \pm 23 \mathrm{vs} 16 \pm 3 \mathrm{~ms}$ at baseline, $\mathrm{p}<0.05)$ paw. $\mathrm{p} 62 \mathrm{KO}$ mice were protected, with adhesive removal times in the affected $(51 \pm 44$ vs $28 \pm 7 \mathrm{~ms}$ at baseline, $\mathrm{p}<0.05)$ and unaffected $(61 \pm 39$ vs baseline $28 \pm 7 \mathrm{~ms}$ at baseline) paws substantially improved when compared to WT mice at 72 hour.

Conclusions Our findings show that p62 KO mice are protected from cerebral ischaemia. This protection persists despite p62 KO mice being glucose intolerant, suggesting that p62 may be a meaningful therapeutic target even in comorbid diabetic populations. Further studies are needed to ascertain whether short-term functional recovery afforded by p62 deficiency persists long-term.

\section{EVIDENCE THAT ALTERED REDOX STATUS RESULTS IN KCA3.1 CHANNEL REDUCED ENDOTHELIAL CELL SURFACE EXPRESSION}

${ }^{1}$ Paul Fraser, ${ }^{1}$ Anas Bani Khalaf, 'Giovanni Mann, 'Matthew Smith, ${ }^{2}$ Geraldine Clough. ${ }^{1}$ King's College London; ${ }^{2}$ University of Southampton, UK

10.1136/postgradmedj-2018-fpm.21

Introduction Endothelial derived hyperpolarization $(\mathrm{EDH})$ is an important path to vasodilatation especially in small arteries, and is more important in women and the elderly. Our previous work has shown that this becomes impaired in mice that had experienced a high fat diet during gestation up to weaning, with concomitant reduction in endothelial surface KCa3.1 expression. ${ }^{1}$ We have tested the idea that altered redox status affects the location of $\mathrm{KCa} 3.1$ in human dermal microvascular endothelial cells (HDMECs).

Methods HDMECs were obtained from PromoCell (Heidelberg, Germany) and cultured in MV2 growth medium containing $1 \%$ penicillin-streptomycin in $5 \% \quad \mathrm{O}_{2}, 5 \% \mathrm{CO}_{2}$ at $37^{\circ} \mathrm{C}$ to passage 6 and 7 in the SCI-tive Physiological Oxygen Workstation from Baker Ruskinn (Maine, USA) for at least 5 days before experiments. The cells were plated on fibronectin (10 $\mu \mathrm{g} . \mathrm{ml}^{-1}$ ) coated 8 well micro-slides (Ibidi, Martinsried, Germany) and incubated for 48 hour, treated with PBS or $\mathrm{H}_{2} \mathrm{O}_{2} 100 \mu \mathrm{M}$ in PBS for $10 \mathrm{~min}$., and fixed with $4 \%$ formalin. Immunofluorescence was performed by incubating with anti-KCa3.1 mouse monoclonal antibody (AL-051 Alomone, Israel) at $4^{\circ} \mathrm{C}$ overnight, with half of the wells being treated with $2 \%$ Triton-X to permeablize the cells. The Alexa 488 labelled anti-mouse antibody for 1 hour and Texas Red Lycopersicon Esculentum Lectin (to stain the cell surface) for $20 \mathrm{~min}$, were applied to the cells at room temperature and finally the DAPI nuclear stain was applied for $3 \mathrm{~min}$. The cells were examined using a x60 1.3 NA oil-immersion objective and images captured via a Hamamatsu RG3 camera into a computer. Using ImageHopper software and computer-driven focussing device (Prior Instruments, UK) we took 10 serial images of $\mathrm{KCa} 3.1$ at $1.0 \mu \mathrm{m}$ intervals, which were integrated using Image J. These images were analysed for the fractional area of an endothelial cell occupied by $\mathrm{KCa} 3.1$, defined as bright spots between 1 to $4 \mu \mathrm{m}$ diameter in the relevant fluorescence channel.

Results The density of channels on the surface, defined as the integrated image of non-permeablized KCa3.1 channels, was $9.5 \pm 0.68 \mu \mathrm{m}^{-2} \times 10^{3}$ (mean \pm sem), while in the permeablized cells the density within the whole cell area was 13.9 $\pm 1.89 \mu \mathrm{m}^{-2} \times 10^{3}(\mathrm{p}<0.05$, ' $\mathrm{t}$ ' test, 4 experiments with 20 cells per experimental group) indicating that about $70 \%$ of the channels normally reside on the cell surface. Treatment with $\mathrm{H}_{2} \mathrm{O}_{2}$ had little effect on the total cell density (reduced 
to $12.3 \pm 1.56$ ), but reduced the surface density to $5.25 \pm 0.47$ $(\mathrm{p}<0.01)$ i.e. reduced to $43 \%$.

Conclusion Thus even a brief exposure to oxygen stress will result in the diminution of $\mathrm{KCa} 3.1$ on endothelial surface, and this may have implications for the ability of arterioles to dilate.

\section{REFERENCE}

1. Stead R, et al. J Hypertens 2016:34:452-63.

\section{UPTICK/DOWNTICK - THE FUTURE OF MORBIDITY AND MORTALITY MEETINGS}

${ }^{1}$ Helen Walker, 'Steve Harris, ${ }^{2}$ Aasiyah Rashan. ${ }^{1}$ University College Hospital; ${ }^{2}$ INFORM project

\subsection{6/postgradmedj-2018-fpm.22}

Introduction Morbidity and mortality ( $\mathrm{M}$ and $\mathrm{M}$ ) meetings are a national requirement for all departments in hospital. While they should confer improvement in education and patient care, they often 'lack defined structure, resource support and...heterogeneity in case evaluation'. ${ }^{1}$ UK critical care has an embedded routine audit data collection on outcomes which is exported to a central body, Intensive Care National Audit and Research Centre (ICNARC), for cleaning and analysis. This data is submitted monthly but is returned on a quarterly basis preventing the timely identification of deaths or survivals that are unexpected (based on case-mix). In critical care, where the expected mortality is high, attention is fatigued if all mortality is reviewed and a more specific approach is required.

A fundamental prerequisite for a good $\mathrm{M}$ and $\mathrm{M}$ meeting is appropriate, targeted case selection. Currently, time is wasted reviewing cases that are selected without systematic focus on 'important variation', so contemporaneous feedback on care is lost and learning and engagement are diminished. To combat this, we have developed a timely, targeted and transformative $\mathrm{M}$ and $\mathrm{M}$ meeting based on data-driven case selection.

Methods

- Using code in $\mathrm{R}$ to generate an automated variable lifeadjusted display (VLAD) chart using near real-time routine data from our electronic health record. This reveals the high yield outlying 'uptick/downtick' patients (patients who survived and should have died and vice-versa) (figure 1).

- Identifying crucial learning points from the clinical documentation of those selected 'uptick/downtick' patients using a specially developed Intensive Care Unit Structured Case Note Judgement Review format.

- Presentation of these targeted cases in a structured multidisciplinary setting with key personnel highlighting the impact of timely narrative feedback, promoting staff engagement and morale and encouraging the growth of a proactive culture of safety and quality.

Results The VLAD cumulatively charts the effect of each individual admission using the ICNARC generated probability of death. Each death represents a 'downtick' in the plot and each survivor represents an 'uptick'. When a patient predicted to survive dies, then the downtick will be prominent (conversely a patient who survives despite a high predicted mortality will make a prominent uptick).

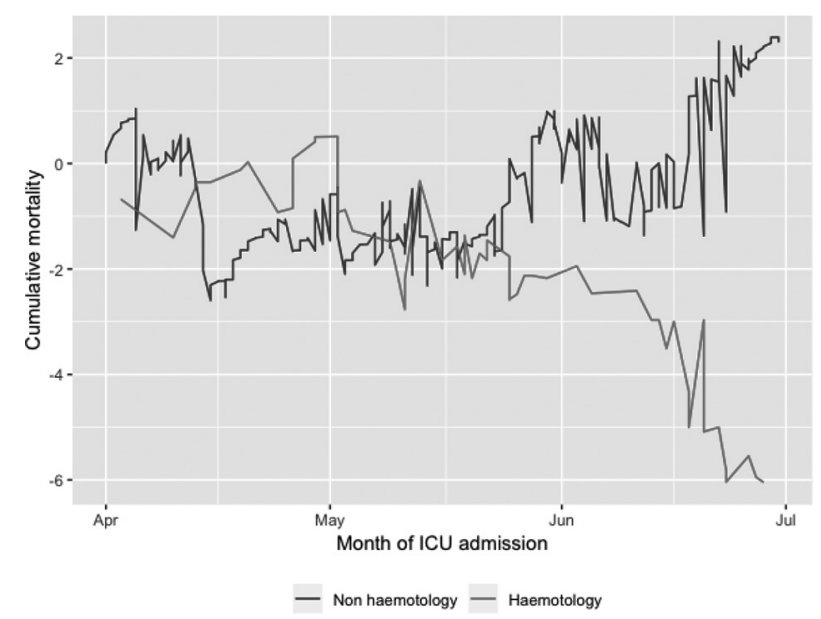

Abstract 11 Figure 1 A VLAD to show haematology and nonhaematology mortality on ICU

Conclusion Our $\mathrm{M}$ and $\mathrm{M}$ model is an original in-depth and specific data mine of critical care patient morbidity and mortality. This unique process seeks to provides an enabled and focussed method of case selection and emphasise the potential marginal gains that can be made in patient care. Over time, it will improve future clinical decision making and therefore patient outcomes.

\section{REFERENCE}

1. Vreugdenburg, et al. Morbidity and mortality meetings: gold, silver or bronze? ANZ Journal of Surgery 2018;88:966-974.

\section{POTENTIAL COST-EFFECTIVENESS OF SPECTRAL PHOTON-COUNTING COMPUTED TOMOGRAPHY (SPCCT) VERSUS CT COMBINED WITH CT ANGIOGRAPHY (CTA) IN THE IDENTIFICATION AND TREATMENT OF ISCHAEMIC STROKE PATIENTS IN THE UK}

Anne Claire Peultier, Ken Redekop, Hans Severens. ESHPM Erasmus University, Rotterdam, The Netherlands

\subsection{6/postgradmedj-2018-fpm.23}

Introduction In current care, eligibility for thrombolysis or mechanical thrombectomy as acute stroke treatments is based on imaging (CT and CTA), time since onset and patient history. Thrombectomy will be routinely commissioned by NHS England provided it can be achieved within 6 hours of the onset of stroke. In case advanced imaging indicates salvageable brain tissue beyond the 6 hour window, thrombectomy will be commissioned up to 12 hours after stroke onset. SPCCT is an emerging technology expected to have an increased resolution. Besides identifying ischaemic stroke patients, SPCCT is expected to provide information about the presence of salvageable brain tissue and, in turn, identify those who are likely to benefit from thrombectomy beyond 6 hours. The aim of this study was to investigate the potential cost-effectiveness of SPCCT (versus CT +CTA) in identifying and treating ischaemic stroke patients in the UK.

Methods A decision-analytic model built on a decision tree and a Markov trace was developed. A hypothetical cohort of suspected stroke patients aged 74 was modeled (first stroke ever). Costs, outcomes and probabilities were obtained from the literature. Outcomes of the model included costs, life- 